\title{
AOR
}

Selected Papers of \#AolR2021:

The 22nd Annual Conference of the Association of Internet Researchers Virtual Event / 13-16 Oct 2021

\section{THE GOVERNANCE OF FACEBOOK PLATFORM}

\author{
Anne Helmond \\ University of Amsterdam \\ Fernando N. van der Vlist \\ Utrecht University and University of Siegen \\ Marcus Burkhardt \\ University of Siegen \\ Tatjana Seitz \\ University of Siegen
}

\section{Introduction}

Policymakers and competition and regulation authorities worldwide recognise application programming interfaces (APIs) for their role in processes of datafication and platformisation; even as a way to 'dominate the digital world' (Iyer and Getchell, 2018; van Dijck, 2020). APIs serve as the 'lingua franca for the exchange of data and services between companies' (lyer and Getchell, 2018) and are of strategic importance for platform companies like Google and Facebook as the web became more data-intensive with the rise of the platform as its dominant technological and business model. APIs have become the core elements of digital infrastructure that underpin today's vibrant platform ecosystems and the platform economies and societies that depend on them. Consequently, lyer and Getchell warn that regulators should not only focus on the market dominance of platform companies but also on their 'data dominance' specifically, how platform companies use APIs to share data or insights with third parties. Competition authorities and regulators in Europe and in the USA increasingly scrutinise anti-competitive uses and potential data misuses centred around Facebook's APIs and the platform's monopoly power. Despite broad recognition of the importance of APIs, we lack a comprehensive understanding of them as complex technical objects. As such, critical scholars argue that '[r]egulatory fixes require detailed insights into how technology and business models work' (van Dijck et al., 2018: 158) and call for the 
'observability' of platforms as an explicit means of regulation (Rieder and Hofmann, 2020).

\section{Approach}

We present the results of an empirical case study of the structure and evolution of Facebook's APIs and their relation to platform governance to highlight the technicity that is, the technical dimension and dynamics - of how and what platforms like Facebook seek to govern. The analysis is focused on Facebook's APIs, which have been among the most popular, widely used, and most controversial APIs for over a decade (Albright, 2018). While the Facebook Graph API (GAPI) is the best-known, there are hundreds of distinct Facebook API entities that have (dis)appeared over the years. We explore how these APIs have changed or evolved in the face of public controversy and mounting criticism around Facebook, and what this might reveal about governance by large platforms like Facebook.

Specifically, we consider the relationality between the design of Facebook's APIs, platform governance, and (data) strategy from a material-evolutionary perspective on three levels: (1) the structure of Facebook's entire API architecture, (2) core API objects in terms of their properties, connections, and parameters, and (3) their associated permissions, as handled through Facebook applications and Login, using current and archived Facebook developer pages. On the level of the API architecture, we derived the link structure of 63,027 reference documentation pages that describe Facebook's APIs. On the level of individual API objects, we examine one of the core (and most connective) nodes in the entire reference documentation: the Graph API User object. Finally, we examine application permissions, which represent a gateway for apps to access data from Facebook, and which have become an increasingly important governance mechanism.

The empirical analysis is based on a large corpus of developer pages as retrieved from the 'live' web and from the Internet Archive Wayback Machine. We downloaded 3,394 'live' web pages from developers.facebook.com and retrieved 1,960,901 developer pages from the Wayback Machine, going back to the initial Facebook API (beta) launch (August 2006 - February 2020). Because Facebook does not provide an archive of its developer website, these independent archived sources provide an important means for the observability of platforms (Helmond and van der Vlist, 2019). Additionally, we consult Facebook's own Developer Blog and News sections, as well as external technology journalism blogs, interviews and testimonies by CEO Mark Zuckerberg, and 7,000 pages of documents leaked during Facebook's litigation with app developer Six4Three in California state court. We thus draw on a variety of primary Facebook sources and external sources that provide important contextual information about specific changes.

\section{Findings}

We find that Facebook's APIs have evolved from a simple programming interface for development into a complex layered and interconnected governance arrangement, wherein technical API specifications serve to enforce (changes to) platform policy and 
(data) strategy. We thus contend that governance by platforms is about more than a platform's content moderation policy, terms and conditions, and corporate governance structure; instead, it is also the design (and redesign) of technical API specifications that condition and control the possibilities for the exchange of data and services between software systems and organisations. As such, this study contributes to the ongoing debate on platform governance within the platform studies literature (e.g. Caplan and Gillespie, 2020; Gillespie, 2018; Gorwa, 2019). We argue that it is important to study the technicity (and materiality) of governance by platforms like Facebook to understand the sources of their infrastructural power, and how that power enables them 'to shift the economic dynamics of competition and monopolization in their favor' (Blanke and Pybus, 2020: 2).

To understand how the dependence relationships between a platform like Facebook and its ecosystem are established and governed, it is important to track changes in the dynamics between a platform's API design (or technical architecture), governance, and strategy in relation to public controversy, or other social and legal pressure to change. Web archives serve an important role in providing a means for the observability of platforms by preserving the material traces of platform evolution.

\section{References}

Albright J (2018) The Graph API. Medium: Tow Center, March 21. Available at: https://medium.com/tow-center/the-graph-api-key-points-in-the-facebook-andcambridge-analytica-debacle-b69fe692d747.

Blanke T and Pybus J (2020) The material conditions of platforms: Monopolization through decentralization. Social Media + Society 6(4). DOI:

10.1177/2056305120971632.

Caplan R and Gillespie T (2020) Tiered governance and demonetization: The shifting terms of labor and compensation in the platform economy. Social Media + Society. DOI: 10.1177/2056305120936636.

Gillespie T (2018) Regulation of and by Platforms. In: Burgess J, Poell T, and Marwick A (eds) The SAGE Handbook of Social Media. London: SAGE Publications, pp. 196212.

Gorwa R (2019) What is platform governance? Information, Communication \& Society 22(6): 854-871. DOI: 10.1080/1369118X.2019.1573914.

Helmond A and van der Vlist FN (2019) Social Media and Platform Historiography: Challenges and Opportunities. TMG - Journal for Media History 22(1): 6-34. DOI: 10.18146/tmg.434/. 
lyer B and Getchell K (2018) Why APIs should be regulated. MIT Sloan Management Review, February 13. Available at: https://sloanreview.mit.edu/article/why-regulatedigital-organizations-apis/.

Rieder B and Hofmann J (2020) Towards platform observability. Internet Policy Review 9(4). Available at: https://policyreview.info/articles/analysis/towards-platformobservability.

van Dijck J (2020) Seeing the forest for the trees: Visualizing platformization and its governance. New Media \& Society. DOI: 10.1177/1461444820940293.

van Dijck J, Poell T and de Waal M (2018) The Platform Society. Oxford University Press. Available at: https://doi.org/10.1093/oso/9780190889760.001.0001. 